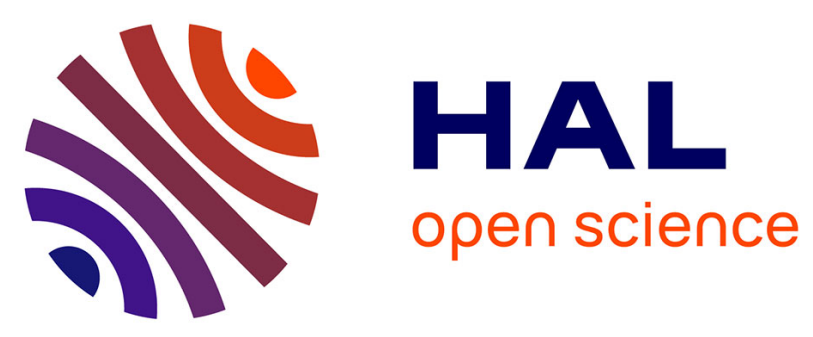

\title{
Nonsmooth modal analysis: Investigation of a 2-dof spring-mass system subject to an elastic impact law
}

Anders Thorin, Mathias Legrand, Stéphane Junca

\section{To cite this version:}

Anders Thorin, Mathias Legrand, Stéphane Junca. Nonsmooth modal analysis: Investigation of a 2dof spring-mass system subject to an elastic impact law. International Design Engineering Technical Conferences \& Computers and Information in Engineering Conference, ASME, Aug 2015, Boston, United States. 10.1115/DETC2015-46796 . hal-01185973

\section{HAL Id: hal-01185973 \\ https://hal.science/hal-01185973}

Submitted on 25 Nov 2016

HAL is a multi-disciplinary open access archive for the deposit and dissemination of scientific research documents, whether they are published or not. The documents may come from teaching and research institutions in France or abroad, or from public or private research centers.
L'archive ouverte pluridisciplinaire HAL, est destinée au dépôt et à la diffusion de documents scientifiques de niveau recherche, publiés ou non, émanant des établissements d'enseignement et de recherche français ou étrangers, des laboratoires publics ou privés. 


\title{
Nonsmooth modal analysis: investigation of a 2-dof spring-mass system subject to an elastic impact law
}

\author{
Anders Thorin ${ }^{1 *}$, Mathias Legrand ${ }^{1}$, Stéphane Junca $^{2}$
}

\begin{abstract}
The well-known concept of normal mode for linear systems has been extended to the framework of nonlinear dynamics over the course of the 20th century, initially by Lyapunov, and later by Rosenberg and a growing community of researchers in modal and vibration analysis. This effort has mainly targeted nonlinear smooth systems - the velocity is continuous and differentiable in time-even though systems presenting nonsmooth occurrences have been increasingly studied in the last decades to face the growing industrial need of unilateral contact and friction simulations. Yet, these systems have nearly never been explored from the standpoint of modal analysis.

This contribution addresses the notion of modal analysis of nonsmooth systems. Developments are illustrated on a seemingly simple 2-dof autonomous system, subject to unilateral constraints reflected by a perfectly elastic impact law. Even though friction is ignored, its dynamics appears to be extremely rich. Periodic solutions are sought for given numbers of impacts per period and nonsmooth modes are illustrated for one and two impacts per period in the form of two-dimensional manifolds in the phase space. Also, an unexpected bridge between these modes in the frequency-energy plots is observed.
\end{abstract}

\section{Keywords}

vibro-impact oscillator, nonlinear modes of vibration, nonsmooth modal analysis, autonomous response

${ }^{1}$ Department of Mechanical Engineering, McGill University, Montréal, Québec, Canada

${ }^{2}$ Mathematics Laboratory JAD and Team COFFEE INRIA, Université de Nice Sophia-Antipolis, Nice, France

*Corresponding author: anders.thorin@mcgill.ca

\section{Introduction}

Vibration analysis is easily carried out in the framework of linear elastodynamics because the principle of superposition says that any solution is a combination of linear normal modes. The notion of modes has been extended in the context of nonlinear dynamics [1, 2, 3, 4] which opens doors to efficient dynamic analysis of smooth nonlinear systems in the neighbourhood of fixed points and to model-reduction of large-scale systems [5] Nevertheless, this extension relies on the smoothness of the solutions and many mechanical systems such as industrial robots, rotating machinery may undergo unilateral contact with adjacent components. Nonlinear modes have also been studied on piecewise linear systems with regularized contact occurrences but with no general outcome regarding modal analysis $[6,7,8]$. When not regularized, unilateral contact events induce discontinuities in velocity and usual ordinary differential equations fo not properly capture nonsmooth events such as impacts [9]. Such a system is investigated in the present work.

Nonsmooth systems, i.e. systems undergoing nonsmooth events, have been increasingly studied in the last two decades [9, 10] usually in the time-domain. Vibration analysis of forced nonsmooth systems is reported in the literature (see e.g. [11, 12] among many others). Nevertheless, very few works tackle such systems with no external periodic forcing, with the aim of identifying nonlinear modes of vibration $[13,14,15,16,17]$. The latter are commonly defined as continuous families - continuity with respect to the energy — of periodic orbits in the phase space. Extensions to nonsmooth systems yield families of nonsmooth periodic orbits. Illustrations are provided on an elementary system with two degrees-of-freedom exhibiting one and two impacts per period (ipp), similar to those studied in $[18,19]$; we highlight again that here, the autonomous system only is investigated.

First, the model is exposed and the expression of the autonomous periodic solutions is derived. It is shown that the existence of periodic solutions depends on three necessary conditions. These conditions are explored for one and two ipp. Continuous families of admissible solutions organized on two-dimensional manifolds are introduced. Nonsmooth modes are then defined and illustrated. Unexpectedly, two supporting manifolds are connected by a "bridge" in the frequency-energy plot: this is described in the last section. Some of the proposed results have to be taken as conjectures since they have only been numerically observed, on a number of calculations, yet not mathematically proven.

The system of interest consists of two springs $k_{1}$ and $k_{2}$ connected to two masses $m_{1}$ and $m_{2}$ in series, as depicted in Figure 1.

The corresponding displacements are denoted $x_{1}(t)$ and $x_{2}(t)$, respectively. For sufficiently large amplitudes, the second mass $x_{2}$ meets a rigid obstacle and thus should satisfy a Signorini condition which prevents any interpenetration with the foundation [20]:

$\forall t \geq 0, \quad\left\{\begin{array}{l}d-x_{2}(t) \geq 0 \\ \lambda(t) \geq 0 \\ \lambda(t)\left(d-x_{2}(t)\right)=0\end{array}\right.$

where $\lambda$ is the reaction force of the obstacle on the second mass, negative for sticking. Note that the first mass is not subject to any unilateral contact condition.

To ensure the well-posedness of the problem, information regarding the impact must be added [21]. In this work, this information is incorporated in the form of a perfectly elastic Newton impact law through the introduction of a restitution coefficient $1 \geq e \geq 0$. The dynamics is governed by the following equations:

$$
\begin{aligned}
& \forall t \text { such that } x_{2}(t)<d \\
& \qquad\left[\begin{array}{cc}
m_{1} & 0 \\
0 & m_{2}
\end{array}\right]\left(\begin{array}{l}
\ddot{x}_{1}(t) \\
\ddot{x}_{2}(t)
\end{array}\right)+\left[\begin{array}{cc}
k_{1}+k_{2} & -k_{2} \\
-k_{2} & k_{2}
\end{array}\right]\left(\begin{array}{l}
x_{1}(t) \\
x_{2}(t)
\end{array}\right)=\left(\begin{array}{l}
0 \\
0
\end{array}\right)
\end{aligned}
$$

$\forall t_{i}$ such that $x_{2}\left(t_{i}\right)=d$,

$$
\dot{x}_{2}\left(t_{i}^{+}\right)=-e \dot{x}_{2}\left(t_{i}^{-}\right)
$$




\begin{tabular}{|c|c|c|c|}
\hline \multicolumn{2}{|c|}{ Nomenclature } & $\tilde{\mathbf{q}}$ & vector of modal coordinates and modal velocities \\
\hline & & $\Delta t$ & time-step \\
\hline$\Omega$ & diagonal matrix of the frequencies & $\dot{x}_{1}, \dot{x}_{2}$ & velocities of the masses \\
\hline $\mathbf{0}_{n}$ & zero matrix of $\mathbb{R}^{n \times n}$ & $\lambda$ & reaction force of the obstacle on the second mass \\
\hline $\mathbf{D}_{1}, \mathbf{D}_{2}$ & eigenvectors of $\mathbf{M}^{-1} \mathbf{K}$ & $\omega_{1}, \omega_{2}$ & eigenvalues of $\mathbf{M}^{-1} \mathbf{K}$ \\
\hline $\begin{array}{l}\mathbf{I}_{n} \\
\mathbf{K}\end{array}$ & $\begin{array}{l}\text { identity matrix of } \mathbb{R}^{n \times n} \\
\text { stiffness matrix }\end{array}$ & $d$ & $\begin{array}{l}\text { distance between resting position of second mass and } \\
\text { obstacle }\end{array}$ \\
\hline M & mass matrix & $e$ & coefficient of restitution \\
\hline $\mathbf{N}$ & mapping of the pre-impact velocities to the post-impact & $k_{1}, k_{2}$ & stiffnesses of the springs \\
\hline & velocities, on $\dot{\mathbf{x}}$ & $m$ & number of impact(s) per period \\
\hline $\begin{array}{l}\mathbf{P} \\
\tilde{\mathbf{N}}\end{array}$ & change of basis matrix, in $\mathbb{R}^{2 \times 2}$ & $m_{1}, m_{2}$ & masses \\
\hline & velocities, on $\tilde{\mathbf{x}}$ & $\begin{array}{l}1 \\
t\end{array}$ & $\begin{array}{l}\text { period } \\
\text { time }\end{array}$ \\
\hline$\tilde{\mathbf{S}}$ & $\begin{array}{l}\text { mapping of initial conditions to current state in a free } \\
\text { phase }\end{array}$ & $\begin{array}{l}T_{1}, T_{2} \\
t_{i}\end{array}$ & $\begin{array}{l}\text { modal periods, } T_{i}=2 \pi / \omega_{i} \\
\text { impact time instant, } i \in\{1, \ldots, m\}\left(t_{1}=0 \text { by choice }\right)\end{array}$ \\
\hline $\mathbf{q}$ & modal coordinates & $x_{1}, x_{2}$ & displacements of the masses \\
\hline $\mathbf{x}$ & vector of the displacement $x_{1}$ and $x_{2}$ (nodal coordinates) & $:=$ & equals, by definition \\
\hline
\end{tabular}

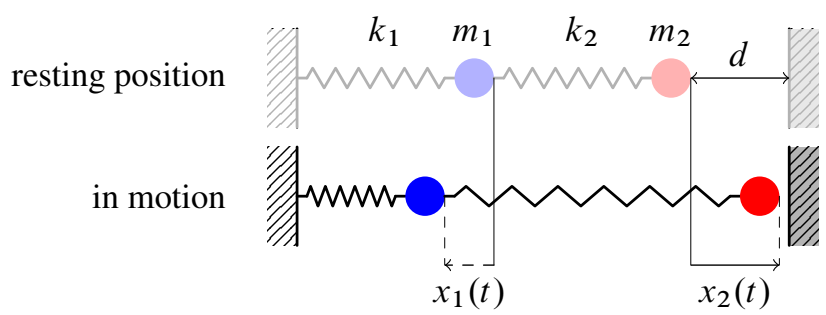

Figure 1. Model of interest and notations.

where $x_{1}$ is twice differentiable everywhere in time and $x_{2}$ twice differentiable almost everywhere (i.e. everywhere except for $t_{i}$ such that $\left.x_{2}\left(t_{i}\right)=d\right)$ in time. The two quantities $\dot{x}_{2}\left(t_{i}^{-}\right)$and $\dot{x}_{2}\left(t_{i}^{+}\right)$stand for the velocity of the second mass just before and just after the impact, respectively-more precisely the left- and right-hand side limits of $\dot{x}_{2}$ in $t_{i}$.

In this paper, periodic orbits are solely targeted. To ease the discussion to come, problem (2) together with periodicity are written in a slightly different manner. The mathematical problem of interest becomes: Given a number of impacts $m \in \mathbb{N}^{\star}$, find the two displacements $x_{1}(\cdot)$ and $x_{2}(\cdot)$, one period $T \in \mathbb{R}_{+}^{\star}$ and $m$ intermediate impact time instants $t_{1}, \ldots, t_{m}$ such that:

$\forall t \in \mathbb{R}_{+}^{\star}, t \notin\left\{t_{1}, \ldots, t_{m}\right\}$, Equation (2a) is satisfied

$\forall t_{i} \in\left\{t_{1}, \ldots, t_{m}\right\}, \dot{x}_{2}\left(t_{i}^{+}\right)=-e \dot{x}_{2}\left(t_{i}^{-}\right)$

$\forall t \geq 0, x_{1}(t+T)=x_{1}(t)$ and $x_{2}(t+T)=x_{2}(t)$

$\forall t_{i} \in\left\{t_{1}, \ldots, t_{m}\right\}, x_{2}\left(t_{i}\right)=d$

$\forall t \in\left[0 ; T\left[, x_{2}(t)=d \Longrightarrow t \in\left\{t_{1}, t_{2}, \ldots, t_{m}\right\}\right.\right.$

Equation (3a) ensures the solutions obey the dynamics; (3b) is the impact law; $(3 \mathrm{c})$ guarantees the periodicity of the solution; ( $3 \mathrm{~d}$ ) ensures that $t_{1}, \ldots, t_{m}$ are actually impact instants, and finally (3e) means that $x_{2}$ does not undergo more than $m$ impacts on the time interval $[0 ; T$. Without any loss of generality, the first impact is now assumed to occur at $t_{1}=0$. By periodicity, this also corresponds to an impact at $T$, which is the first impact of the second period.

The periodicity ( $3 \mathrm{c}$ ) requires that $e=1$, which is considered here. Indeed, $e<1$ leads to an instantaneous loss of kinetic energy of the second mass and therefore an instantaneous change of the total energy of the system at each impact.

With the final objective of calculating solutions to system (3), necessary conditions on their existence are now derived. This is achieved as follows: derive the free dynamics on $] 0 ; t_{2}[$, enforce the impact law at $t_{2}$, and repeat with the subsequent sequences of free dynamics and corresponding impact occurrences up to the last interval $] t_{m} ; T[$. This yields a necessary condition on the pe$\operatorname{riod} T$ and the intermediate impact time instants $t_{2}, \ldots, t_{m}$. The linear dynamics is governed by Eq. (2a) which can be compactly cast in a matrix form:

$\mathbf{M} \ddot{\mathbf{x}}+\mathbf{K x}=\mathbf{0}$

with $\mathbf{x}(t)=\left(x_{1}(t) x_{2}(t)\right)^{\top}$. The pairs of eigenvalues and eigenvectors of $\mathbf{M}^{-1} \mathbf{K}$ are denoted by $\left(\omega_{1}, \omega_{2}\right)$ and $\left(\mathbf{D}_{1}, \mathbf{D}_{2}\right)$, respectively. The two corresponding periods are given by $T_{1}=2 \pi / \omega_{1}$ and $T_{2}=2 \pi / \omega_{2}$. Introducing $\mathbf{P}=\left[\mathbf{D}_{1}^{\top} \mathbf{D}_{2}^{\top}\right]$ and the vector of modal coordinates $\mathbf{q}(t)=\mathbf{P}^{-1} \mathbf{x}(t)$, Eq. (4) can be diagonalized as

$\mathbf{I}_{2} \ddot{\mathbf{q}}+\mathbf{\Omega} \mathbf{q}=\mathbf{0}$

which gathers two uncoupled equations. In Eq. (5), the notations $\mathbf{I}_{2}=\operatorname{diag}(1,1)$ and $\boldsymbol{\Omega}=\mathbf{P}^{-1} \mathbf{M}^{-1} \mathbf{K P}=\operatorname{diag}\left(\omega_{i}^{2}\right)_{i=1,2}$ are used. This second order differential equation can be transformed into a first order differential equation:

$\left(\begin{array}{c}\dot{\mathbf{q}}(t) \\ \ddot{\mathbf{q}}(t)\end{array}\right)=\left[\begin{array}{cc}\mathbf{0}_{2} & \mathbf{I}_{2} \\ -\boldsymbol{\Omega} & \mathbf{0}_{2}\end{array}\right]\left(\begin{array}{c}\mathbf{q}(t) \\ \dot{\mathbf{q}}(t)\end{array}\right)$

Introducing $\tilde{\mathbf{q}}(t)=\left(\mathbf{q}(t)^{\top} \dot{\mathbf{q}}(t)^{\top}\right)^{\top}$, the solutions of the smooth free motions are thus given by:

$\tilde{\mathbf{q}}(t)=\tilde{\mathbf{S}}(t) \tilde{\mathbf{q}}(0)$

where $\tilde{\mathbf{S}}$ is the exponential of the matrix in (6):

$\tilde{\mathbf{S}}(t)=\left[\begin{array}{cccc}\cos \left(\omega_{1} t\right) & 0 & \sin \left(\omega_{1} t\right) / \omega_{1} & 0 \\ 0 & \cos \left(\omega_{2} t\right) & 0 & \sin \left(\omega_{2} t\right) / \omega_{2} \\ -\omega_{1} \sin \left(\omega_{1} t\right) & 0 & \cos \left(\omega_{1} t\right) & 0 \\ 0 & -\omega_{2} \sin \left(\omega_{2} t\right) & 0 & \cos \left(\omega_{2} t\right)\end{array}\right]$

The dynamics is fully determined by the four initial conditions $\tilde{\mathbf{q}}(0)$. Naturally, the components of $\mathbf{q}$ have the major benefit of being dynamically uncoupled, but the condition of unilateral contact inherently involves the physical coordinate $x_{2}(t)$. There is hence no evident better choice for the basis into which the contact dynamics will be described: both the modal (for the free flight) and the physical basis (for the impact condition) are involved. 
When contact occurs, the impact condition ( $3 \mathrm{~b})$ on $\dot{x}_{2}$ and the continuity of $\dot{x}_{1}$ are expressed in a matrix form as:

$\dot{\mathbf{x}}\left(t^{+}\right)=\mathbf{N} \dot{\mathbf{x}}\left(t^{-}\right) \quad$ with $\quad \mathbf{N}:=\left[\begin{array}{cc}1 & 0 \\ 0 & -e\end{array}\right]$

that is, in terms of modal velocities:

$\dot{\mathbf{q}}\left(t^{+}\right)=\mathbf{P}^{-1} \mathbf{N P} \dot{\mathbf{q}}\left(t^{-}\right)$

Overall, the impact law is written

$\tilde{\mathbf{q}}\left(t^{+}\right)=\tilde{\mathbf{N}} \tilde{\mathbf{q}}\left(t^{-}\right)$

where by definition

$\tilde{\mathbf{N}}:=\left[\begin{array}{cc}\mathbf{I}_{2} & \mathbf{0}_{2} \\ \mathbf{0}_{2} & \mathbf{P}^{-1} \mathbf{N P}\end{array}\right]$

Accordingly, the two possible states of the system in $[0 ; T[$ are:

Free phase on any interval $] t_{i} ; t_{i+1}[$, with $i \in 1, \ldots, m$ :

$$
\tilde{\mathbf{q}}(t)=\tilde{\mathbf{S}}\left(t-t_{i}\right) \tilde{\mathbf{q}}\left(t_{i}^{+}\right)
$$

Impact for any time of impact $t_{i} \in\left\{0, t_{2}, \ldots, t_{m}\right\}$ :

$$
\tilde{\mathbf{q}}\left(t_{i}^{+}\right)=\tilde{\mathbf{N}} \tilde{\mathbf{q}}\left(t_{i}^{-}\right)
$$

It is now possible to describe the periodic solutions of period $T$ with $m$ intermediate impact occurrences $\left\{t_{2}, \ldots, t_{m}\right\}$ as listed in Tab. 1 . The periodicity condition $(3 \mathrm{c})$ formulated on the modal

\begin{tabular}{c|r}
$t$ & $\tilde{\mathbf{q}}(t)=$ \\
\hline$t \in\left[0 ; t_{2}[\right.$ & $\tilde{\mathbf{S}}(t) \tilde{\mathbf{q}}(0)$ \\
$t \in\left[t_{2} ; t_{3}[\right.$ & $\tilde{\mathbf{S}}\left(t-t_{2}\right) \tilde{\mathbf{N}} \tilde{\mathbf{S}}\left(t_{2}-0\right) \tilde{\mathbf{q}}(0)$ \\
$\vdots$ & $\vdots$ \\
$t \in\left[t_{m} ; T[\right.$ & $\tilde{\mathbf{S}}\left(t-t_{m}\right) \tilde{\mathbf{N}} \tilde{\mathbf{S}}\left(t_{m}-t_{m-1}\right) \cdots \tilde{\mathbf{N}} \tilde{\mathbf{S}}\left(t_{2}-0\right) \tilde{\mathbf{q}}(0)$
\end{tabular}

Table 1. Modal displacement $\tilde{\mathbf{q}}$ as a piecewise function of time.

coordinates, that is $\tilde{\mathbf{q}}(T)=\tilde{\mathbf{q}}(0)$, can now be expressed as

$\tilde{\mathbf{S}}\left(t-t_{m}\right) \tilde{\mathbf{N}} \tilde{\mathbf{S}}\left(t_{m}-t_{m-1}\right) \cdots \tilde{\mathbf{N}} \tilde{\mathbf{S}}\left(t_{2}-0\right) \tilde{\mathbf{q}}(0)=\tilde{\mathbf{q}}(0)$

or equivalently

$\tilde{\mathbf{q}}(0) \in \operatorname{ker} \mathbf{A}_{m}$

with

$\mathbf{A}_{m}:=\mathbf{S}\left(T-t_{m}\right) \tilde{\mathbf{N}} \mathbf{S}\left(t_{m}-t_{m-1}\right) \cdots \tilde{\mathbf{N}} \mathbf{S}\left(t_{2}-0\right)-\mathbf{I}_{4}$

This $4 \times 4$ matrix $\mathbf{A}_{m}$ stems from Eq. (3a), (3b), and (3c) and governs the existence of periodic solutions with $m$ impact(s) per period. It embeds the free dynamics on every time interval ] $0 ; t_{2}[, \ldots,] t_{m} ; T$ [ and the change of sign of $\dot{x}_{2}$ at impact times $0, t_{2}, \ldots, t_{m}$. It is essential to find impact times $\left\{t_{2}, \ldots, t_{m}\right\}$ for which solutions to (3) may exist. Indeed, if $\operatorname{det} \mathbf{A}_{m} \neq 0$, the unique solution of Eq. (16) is $\tilde{\mathbf{q}}(0)=\left(\begin{array}{llll}0 & 0 & 0 & 0\end{array}\right)^{\top}$. This indicates that $\operatorname{det} \mathbf{A}_{m}=0$ (or equivalently $\operatorname{dim}\left(\operatorname{ker} \mathbf{A}_{m}\right) \geqslant 1$ ) is a necessary condition for the existence of solutions of (3). It is denoted by (NC1).

However, $\mathbf{A}_{m}$ does not include (3d) and (3e). Hence, identifying a period $T$ and impact times $\left\{t_{2}, \ldots, t_{m}\right\}$ using (NC1) does not ensure that the corresponding displactement is a solution of (2). Two additional verifications have to be performed: first, impacts must occur when the second mass meets the obstacle (3d). This condition ensures that the impact law on $x_{2}\left(t_{i}\right)$ is enforced only when $x_{2}\left(t_{i}\right)=d$. Otherwise, the second mass should free-flight in $\left[0 ; T\right.$ [ for times other $0, t_{2}, \ldots, t_{m} \mathrm{n}$ that is $x_{2}<d$ since $x_{2}$ is continuous on $\mathbb{R}^{+}$. Formally and all together these conditions are:

$$
\begin{aligned}
& \text { (NC1) } \operatorname{det}\left(\mathbf{A}_{2}\right)=0 \text { for } T \text { and } t_{2}, \ldots, t_{m} \\
& \text { (NC2) } \forall t \in\left\{0, t_{2}, \ldots, t_{m}\right\}, x_{2}\left(t_{i}\right)=d \\
& \text { (NC3) } \forall t \geq 0, x_{2}(t) \leq d .
\end{aligned}
$$

To illustrate the necessity of (NC2) and (NC3), a displacement $x_{2}(t)$ calculated using $\mathbf{A}_{2}$ and Tab. 1 is represented in Figure 2. It is clear that it is not a solution of (3), because it does not satisfy (NC2) nor (NC3).

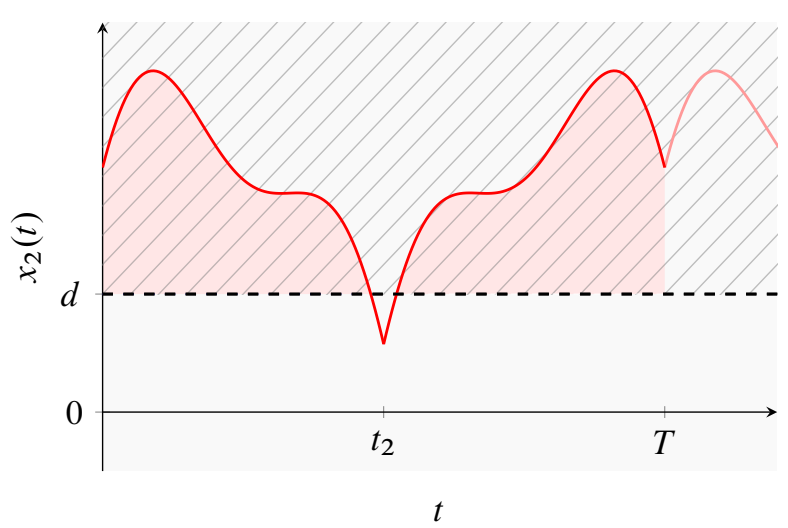

Figure 2. Non-admissible displacement with $m=2:[-] x_{2}(t)$. The motion satisfies condition (NC1) but neither $(N C 2)\left(x_{2}\left(t_{2}\right) \neq\right.$ d) nor (NC3) (because $x_{2} \geq d$ for some $t \in[0 ; T[$ ).

Defining

$\tilde{\mathbf{P}}:=\left[\begin{array}{ll}\mathbf{0}_{2} & \mathbf{P} \\ \mathbf{0}_{2} & \mathbf{P}\end{array}\right]$

condition (NC2) can be expressed in the modal basis as:

$\left(\tilde{\mathbf{P}} \tilde{\mathbf{S}}\left(t_{i}-t_{i-1}\right) \tilde{\mathbf{N}} \tilde{\mathbf{S}} \cdots \tilde{\mathbf{N}} \tilde{\mathbf{S}}\left(t_{2}-0\right) \cdot \tilde{\mathbf{q}}(0)\right) \cdot \mathbf{e}_{2}=d$

$\forall t_{i} \in\left\{t_{2}, \ldots, t_{m}\right\}$. Eq. (19) yields an algebraic system of $m$ equations. It will be shown that whether these equations are redundant or not is an essential criterion for the existence of solutions of (2).

All together, the three necessary conditions yield a necessary and sufficient condition for the existence of a solution to system (3). In terms of computation, (NC3) is the most costly condition because to our knowledge, it can only be checked numerically.

To summarize, given $\left(k_{1}, k_{2}\right),\left(m_{1}, m_{2}\right)$, and $m$ :

1. matrix $\mathbf{A}_{m}$ can be defined as a function of $t_{2}, \ldots, t_{m}, T$.

2. a solution exists if and only if

(NC1) $\operatorname{det}\left(\mathbf{A}_{m}\right)=0$

(NC2) $\forall t \in\left\{0, t_{2}, \ldots, t_{m}\right\}, x_{2}\left(t_{i}\right)=d$

(NC3) $\forall t, d-x_{2}(t) \geqslant 0$

3. when these three conditions are met for some $t_{2}, \ldots, t_{m}, T$, the solution is a piecewise function given by Tab. 1 . 
In the following, the terminology potential solution refers to motions which satisfy conditions (NC1) and (NC2), and admissible solutions refer to motions which satisfy conditions ( $\mathrm{NC} 1),(\mathrm{NC} 2)$, and (NC3), and are thus solutions of (2). Of course, admissible solutions are also potential solutions.

Also, all figures were obtained with $\left(m_{1}, m_{2}\right)=(1,1) \mathrm{kg}$, $\left(k_{1}, k_{2}\right)=(0.85,2) \mathrm{N} \mathrm{m}^{-1}$, and $d=1 \mathrm{~m}$. These values are arbitrary. They correspond to periods $T_{1} \approx 2.97 \mathrm{~s}$ and $T_{2} \approx$ $10.19 \mathrm{~s}$. Units are no longer indicated in the remainder.

\section{Existence of admissible solutions}

\subsection{One impact per period}

In this section, attention is paid to periodic orbits with exactly one impact per period. It can be theoretically shown that the mapping $\mathbf{A}_{1}: T \longmapsto \tilde{\mathbf{N}} \tilde{\mathbf{S}}(T)-\mathbf{I}_{4}$ has a kernel of dimension 1 , for every $T>0$, except for some very specific values of $k_{i}$ and $m_{i}, i=1,2$ corresponding to internal resonances that are not discussed in the present work. Condition (NC1) is thus verified. (NC2) consists in a unique equation:

$\mathbf{P q}(0) \cdot \mathbf{e}_{2}=d \Longleftrightarrow P_{11} q_{1}(0)+P_{12} q_{2}(0)=d$

Since the kernel of $\mathbf{A}_{1}$ is of dimension 1, condition (20) is always satisfied: for the unique vector $\mathbf{y}=\left[y_{1}, y_{2}, y_{3}, y_{4}\right]^{\top}$ in $\operatorname{ker}\left(\mathbf{A}_{1}\right)$, then $\alpha \mathbf{y}$ verifies (NC2) with $\alpha=d /\left(P_{11} y_{1}+P_{12} y_{2}\right)$. Accordingly, there is a unique potential solution for every $T>0$. Let us now focus on (NC3). Given $T$ and a potential solution $x_{2}(t)$, it is possible to calculate numerically its maximum over $[0 ; T]$ and check that $\max x_{2}(t)<d$. It appears that there are intervals of $T$ which contains admissible solutions, as illustrated in Fig. 3. To understand these seemingly random zones of admissible solu-

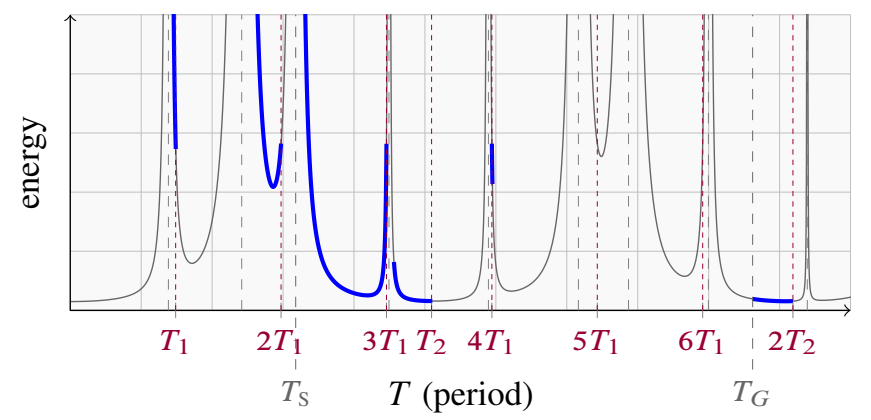

Figure 3. Energies of T-periodic autonous orbits. [-] Potential solutions. [-] Admissible solutions. [- - ] Singular transitions. [--] Linear modal transitions. $T_{S}$ is an example of singular transition. $T_{G}$ is an arbitrary grazing transition used in Fig. 5.

tions, the expression of $x_{2}$ is explored in more details: given an arbitrary $T$ and $t \in[0 ; T[$, its expansion yields:

$$
\begin{aligned}
x_{2}(t)=A(T)\left[B_{1}(T)\right. & \cos \left(\omega_{1}(t-T / 2)\right) \\
+ & \left.B_{2}(T) \cos \left(\omega_{2}(t-T / 2)\right)\right]
\end{aligned}
$$

where

$A(T)=d\left(\omega_{2} P_{12} P_{21} \operatorname{cotan}\left(\omega_{1} T\right)-\omega_{1} P_{11} P_{22} \operatorname{cotan}\left(\omega_{2} T\right)\right)^{-1}$

$B_{1}(T)=P_{12} P_{21} \omega_{2} / \sin \left(\omega_{1} T / 2\right)$

$B_{2}(T)=P_{11} P_{22} \omega_{1} / \sin \left(\omega_{2} T / 2\right)$

from which three kinds of distinct transitions from admissible to non-admissible solutions can be found:
Singular transitions They correspond to singularities of $A(T)$, i.e. solutions $T_{\mathrm{S}}$ to

$\omega_{2} P_{12} P_{21} \operatorname{cotan}\left(\omega_{1} T\right)=\omega_{1} P_{11} P_{22} \operatorname{cotan}\left(\omega_{2} T\right)$

Such $T_{\mathrm{S}}$ are shown in Fig. 3. In the left neighbourhood of a $T_{\mathrm{S}}, x_{2}$ diverges to $+\infty$, while in the right neighbourhood, it diverges to $-\infty$.

Linear modal transitions They may occur when $T$ is a multiple of the linear periods $T_{1}$ or $T_{2}$, that is when

$\omega_{1} T \equiv 0(\bmod 2 \pi) \quad$ or $\quad \omega_{2} T \equiv 0(\bmod 2 \pi)$

Fig. 4 illustrates how an admissible solution smoothly becomes non-admissible. These transitions occur when a linear mode has a sufficiently large energy to graze the wall, level after which they vanish.

Grazing transitions They can be found by comparing $d$ and

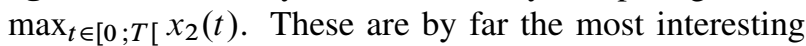
transitions: from 1-ipp trajectories, they lead to multiple impacts per period solutions as shown later. Such a transition period denoted by $T_{\mathrm{G}}$ is represented in Fig. 3 and the corresponding $T_{\mathrm{G}}$-periodic motion is plotted in Fig. 5.

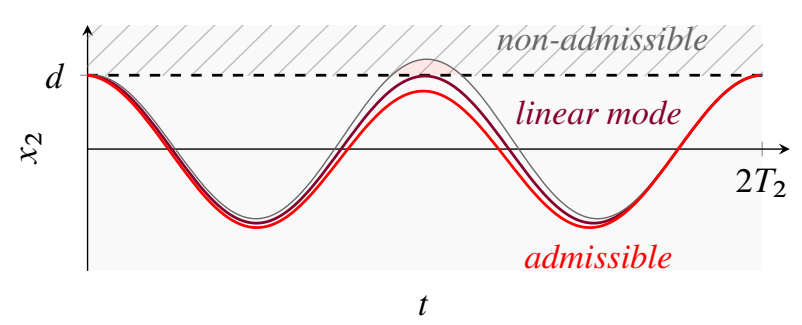

Figure 4. Linear modal transition in the neighbourhood of $2 T_{2}$, for 1-ipp. In the left neighbourhood of $2 T_{1}\left([-] T=2 T_{1}-\varepsilon\right)$, motion is admissible. In the right neighbourhood $([-] T=$ $\left.2 T_{1}+\varepsilon\right)$, it violates the unilateral constraints $\left(x_{2}>d\right)$. The limit case is a modal motion $\left([-] T=2 T_{1}\right)$.

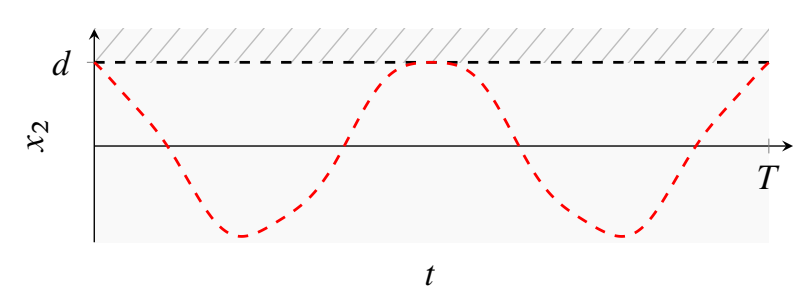

Figure 5. Grazing transition in $T_{G}$ (see Fig. 3). Solutions with two impacts per period emerge from this transition, see last section. $[-]$ Solution for $T=T_{G}$.

Solutions corresponding to a grazing transition always have at least a solution to the equation $x_{2}(t)=d$ in $] 0 ; T[$. They are common solutions to two distinct problems (15) (with the associated conditions (NC1), (NC2), and (NC3)) with a different number of impacts per period $m$. Our observations on numerical examples tend to show that grazing transitions have a very particular property-which we have not proven mathematically yet: the conditions $\forall t_{i}, x_{2}\left(t_{i}\right)=d$ in Eq. (3c) are all redundant. This property has remarkable consequences as explained later. 


\subsection{Two impacts per period}

$T$-periodic admissible solutions with two impacts per period are targeted in this section. To this end, we explore the mapping

$\mathbf{A}_{2}:\left(t_{2}, T\right) \longmapsto \tilde{\mathbf{N}} \tilde{\mathbf{S}}\left(T-t_{2}\right) \tilde{\mathbf{N}} \tilde{\mathbf{S}}\left(t_{2}\right)-\mathbf{I}_{4}$

We recall that the necessary condition $(\mathrm{NC} 1)$ requires that $t_{2}$ and $T$ be such that $\operatorname{det} \mathbf{A}_{2}\left(t_{2}, T\right)=0$. A first result is that given an arbitrary $T$, condition (NC11) is satisfied for a few $t_{2}$ only, if any. This is illustrated in Fig. 6. A second result, which has been

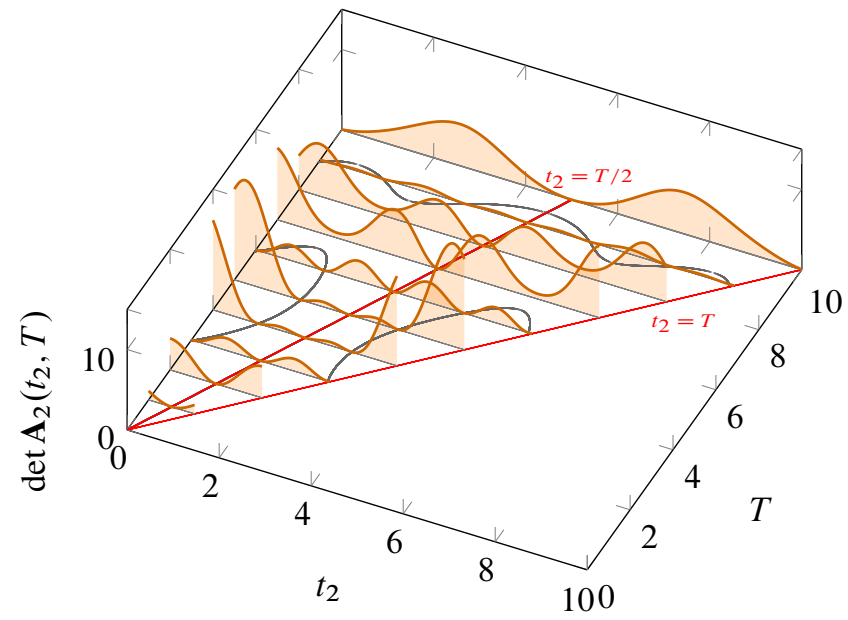

Figure 6. Determinant of $\mathbf{A}_{2}\left(t_{2}, T\right)$ versus $t_{2}$ for several $T$. $[-] \operatorname{det}\left(\mathbf{A}_{2}\left(t_{2}, T\right)\right)$. [-] Roots $\left(t_{2}, T\right)$ of $\operatorname{det}\left(\mathbf{A}_{2}\right)$. For $T \in$ $\{1,2,7,8,10\}$, det $\mathbf{A}_{2}(\cdot, T)$ has no roots $t_{2} \neq T / 2$ and therefore no potential solutions. On the contrary $T \in\{3,4,5,6,9\}$ leads to potential $T$-periodic solutions, some of which can possibly be admissible solutions.

proven but the proof is not detailed for the sake of brevity, is that

$\forall T, \quad \operatorname{det}\left(\mathbf{A}_{2}(T / 2, T)\right)=0$

so that $t_{2}=T / 2$ always satisfies (NC1). This corresponds to 2-ipp $T$-periodic solutions which are in fact 1-ipp $T / 2$-periodic solutions seen on two periods. This result is complemented by a second one which states the existence of a threshold above which there always exists at least a $t_{2} \neq T / 2$ which is root of $\operatorname{det}\left(\mathbf{A}_{2}(\cdot, T)\right)$. Such a threshold, which can be refined in specific cases is $\tilde{T}=3 \pi / \min \left(\omega_{1}, \omega_{2}\right)$ or equivalently $\tilde{T}=$ $3 \max \left(T_{1}, T_{2}\right) / 2$. This can be mathematically written as

$\forall T \geq \tilde{T}, \exists t_{2} \neq T / 2$ such that $\operatorname{det}\left(\mathbf{A}_{2}\left(t_{2}, T\right)\right)=0$

A third interesting result when $m=2$ is that if (NC1) is satisfied, then it is always possible to find initial conditions such that (NC2) is satisfied too. This comes from the following property:

$\operatorname{det}\left(\mathbf{A}_{2}\left(t_{2}, T\right)\right)=0 \quad \Longrightarrow \quad \operatorname{dim}\left(\operatorname{ker}\left(\mathbf{A}_{2}\left(t_{2}, T\right)\right)\right)=2$

Indeed, $\operatorname{dim}\left(\operatorname{ker}\left(\mathbf{A}_{2}\left(t_{2}, T\right)\right)=2\right.$ for some appropriate $\left(t_{2}, T\right)$ means that it is possible to choose the initial conditions such that the motion obeys two independent constraints $x_{2}(0)=d$ and $x_{2}\left(t_{2}\right)=d$.

Again, (NC3) has to be tested numerically and zones of admissible solutions emerge again. This time, they involve both $T$ and $t_{2}$. Numerical results are exposed in Fig. 7. Since $t_{2}=T / 2$ always corresponds to a potential solution for $m=2$, which is nothing else than two periods of the potential solution for $m=1$, configurations with $m=1$ and some of the configurations with $m=2$ are related. This is highlighted in Fig. 8 which combines

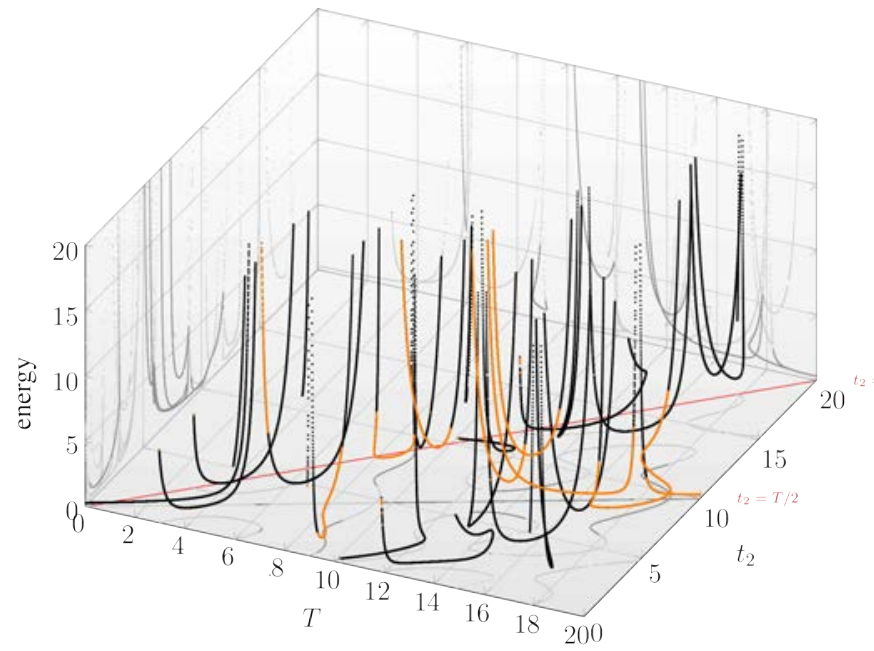

Figure 7. Energies of $T$-periodic 2-ipp motions as a function of $T$ and the time of second impact $t_{2}$. [-] Potential solutions. [-] Admissible solutions.

Fig. 3 and 7 in a two-dimensional graph: the factor 2 dilation of the admissible soltions in the 1-ipp energy plot along the $T$ axis coincides with some parts of the 2-ipp admissible solutions.

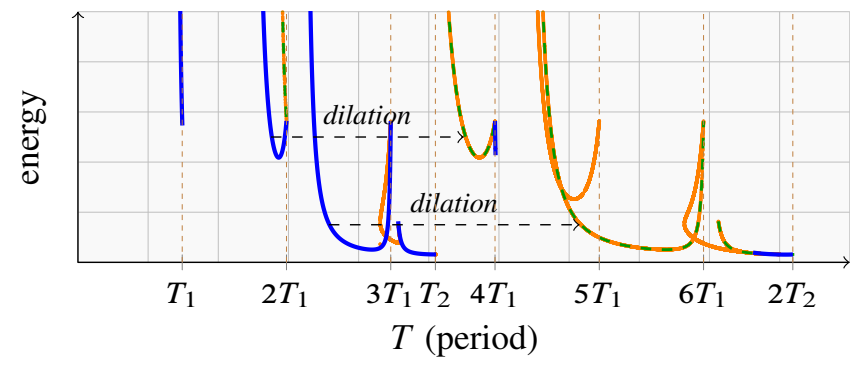

Figure 8. Energies for 1-ipp and 2-ipp orbits. [-] 1-ipp. [-] 2-ipp. [- - ] 2-ipp with $t_{2}=T / 2$. The green curve, which is a subpart of the orange curve, is the factor 2 dilated version of the blue curve along the $T$ axis.

To summarize, when $m=2$, potential solutions of minimal period $T$ sometimes exist when $T$ is smaller than $\tilde{T}$, and always exist when $T \geqslant \tilde{T}$. The existence of potential solutions coincide with the roots of $\operatorname{det}\left(\mathbf{A}\left(t_{2}, T\right)\right)$ which defines continuous curves in the plane $\left(t_{2}, T\right)$. Subparts of these curves define continuous zones of admissible solutions.

\subsection{More than two impacts per period}

More generally, (NC1) seems to be driven by the parity of $m$. The following results have not been mathematically proven but numerically verified on numerous examples. They have therefore to be understood as conjectures:

- If $m$ is odd, then $\operatorname{dim}\left(\operatorname{ker} \mathbf{A}_{m}\right)=1$. (NC2) imposes $m$ conditions, so potential solutions may exist only if these conditions form a system of rank 1, i.e. if they are all equivalent. This seems to happen only for a very few cases corresponding to 1 ipp grazing orbits, see Fig. 9. Any 1-ipp potential solution of period $T / 3$ is of course also a potential solution of a 3 -ipp problem.

- If $m$ is even, then $\operatorname{dim}\left(\operatorname{ker} \mathbf{A}_{m}\right)=2$ and (NC2) imposes $m$ conditions, so potential solutions may exist only if these conditions form a system of rank 1 or 2 . These motions 
have not been thoroughly investigated but no potential solution has been found yet with $m \geq 4$.

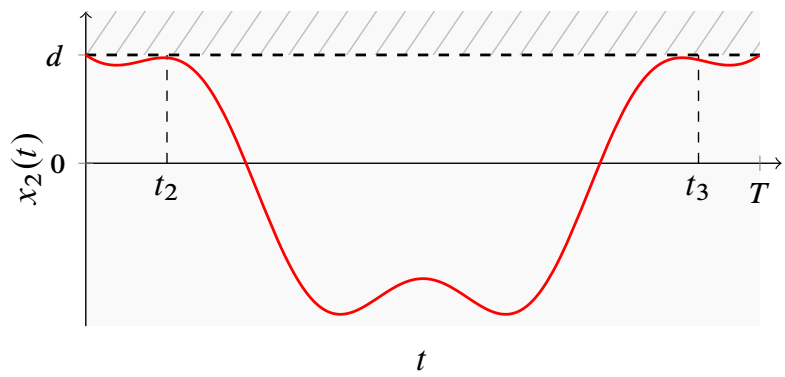

Figure 9. Trajectory with two grazing points and one impact: admissible solution to both the 1-ipp and 3ipp formulations.

\section{Nonsmooth modes of vibration}

In linear dynamics, modes are two-dimensional flat surfaces in the phase space. The notion of normal mode has been particularly extended to smooth nonlinear dynamics in [2], with the following definition: a nonlinear normal mode is a two-dimensional invariant manifold of the phase space, tangent to a mode of the linearized system. Invariant means that if a motion starts on the nonlinear mode at a given time, then it will remain on it for all times. In the same spirit, the definition of a nonsmooth mode of vibration is proposed here: a nonsmooth mode is an invariant manifold formed by a continuum of periodic autonomous (possibly nonsmooth) orbits in the phase space, containing one orbit the a linear mode. For two degree-of-freedom systems, such modes could be described by four functions $f_{1}, \ldots, f_{4}$ of two parameters, e.g. the energy $E$ and time $t$ such that:

$x_{1}=f_{1}(E, t)$

$x_{2}=f_{2}(E, t)$

$\dot{x}_{1}=f_{3}(E, t)$

$\dot{x}_{2}=f_{4}(E, t)$

We showed that for $m=1$, continuous zones of admissible solutions exist. For each of them, it is therefore possible to define two-dimensional surfaces of the phase space $\left(x_{1}, x_{2}, \dot{x}_{1}, \dot{x}_{2}\right)$ which contains all their admissible motions. Note that in the energy-frequency plane, these zones are delimited by $T_{1}, T_{2}$ or one of their multiples, therefore they contain an orbit of a grazing linear mode. Such a surface is represented in Fig. 10. The nonsmooth mode contains one orbit of a grazing linear mode (see red curve in Fig. 10). It is noteworthy that the nonsmooth mode indeed presents some discontinuities on $\dot{x}_{2}$, when $x_{2}=d$. Similarly, nonsmooth modes can be defined for 2-ipp motions, since continua of admissible solutions exist. While 1-ipp nonsmooth modes support periodic orbits of different periods $T$, 2-ipp nonsmooth modes gather periodic orbits which differ in both $t_{2}$ and $T$ - but $t_{2}$ and $T$ cannot vary independently, they are related by the constraint $\operatorname{det}\left(\mathbf{A}_{2}\left(t_{2}, T\right)\right)=0$. To better understand the meaning of the invariant manifold, various $x_{2}$ are displayed in Figure 11. The corresponding orbits are combined in a three-dimensional cross-section $\left(x_{1}, x_{2}, \dot{x}_{2}\right)$ of the phase plot $\left(x_{1}, x_{2}, \dot{x}_{1}, \dot{x}_{2}\right)$, which is an illustration of a cross-section of a 2-ipp nonsmooth mode.

Nonsmooth modes feature numerous benefits. They contain an infinity of admissible solutions which corresponds to a continuum of energy. They provide a picture of the possible admissible

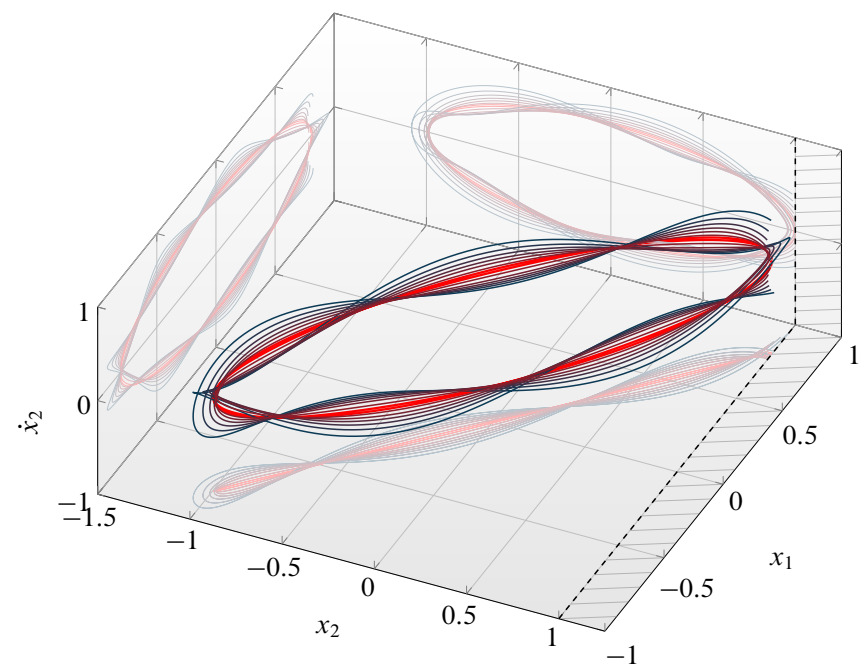

Figure 10. Periodic orbits with one impact per period in the neighbourhood of $2 T_{1}$. These orbits lie on a nonsmooth manifold. [-] Grazing trajectory of the second linear mode. It is visible that $x_{2} \leq 1$, and that when $x_{2}=1, \dot{x}_{2}$ is discontinuous.
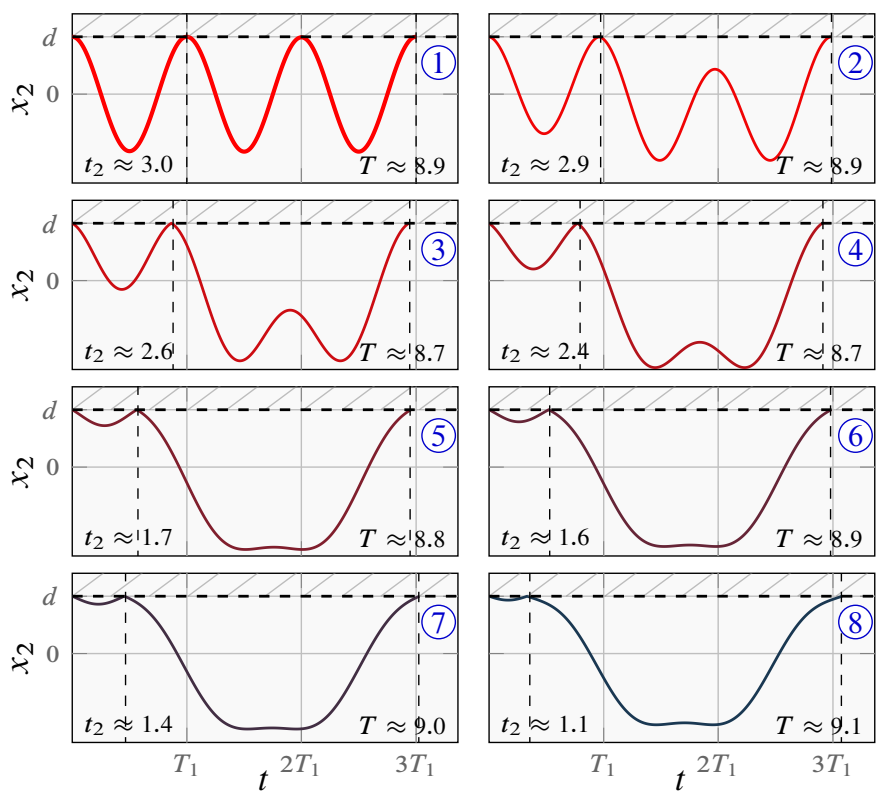

Figure 11. 2-ipp admissible solutions in the neighbourhood of $T=3 T_{1}$. In red, the solution coincides with the linear mode. Other plots obtained by exploring the branch of admissible solutions for different roots $\left(t_{2}, T\right)$ of $\operatorname{det} \mathbf{A}_{2}$. The continuum of orbits is illustrated by the red curve which continuously shades into blue ones.

solutions lying in the phase space of the system of interest. They also provide indications on other regions of the phase space because orbits cannot intersect: for example they can define closed boundaries from which solutions cannot escape. It is also highly possible yet not proved that the associated forced solutions reach high amplitudes when they approach a nonsmooth mode, as it stands in linear and smooth nonlinear dynamics. Additionally, based on preliminary numerical experiments, the developments presented in this paper seem to directly extend to arbitrary $N$ degree-of-freedom systems.

\section{Relation between 1-ipp and 2-ipp nonsmooth modes}

In this last section, a relation between 1-ipp and 2-ipp nonsmooth modes is described. This is not completely understood yet, but 


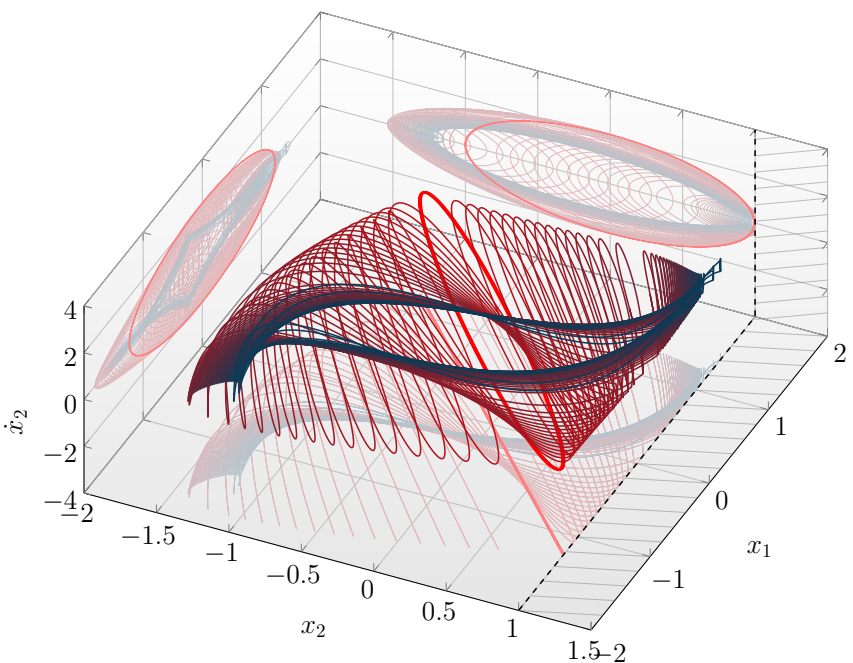

Figure 12. Periodic orbits with two impacts per period in the neighbourhood of $3 T_{1}$. These orbits lie on a nonsmooth manifold. It is visible that $x_{2} \leqslant 1$, and that when $x_{2}=1, \dot{x}_{2}$ is discontinuous. The continuum of orbits is illustrated by the red curve which continuously shades into blue ones.

unexpected admissible solutions exist that were never reported before. Indeed, we recall that for grazing transitions, conditions $x_{2}\left(t_{i}\right)=d, i \in\{1, \ldots, m\}$, are apparently redundant, and can thus reduced to $x_{2}(0)=d$. When $m=2$, for $t_{2}$ and $T$ such that $\operatorname{det}\left(\mathbf{A}_{2}\left(t_{2}, T\right)\right)=0$, then $\operatorname{dim}\left(\operatorname{ker} \mathbf{A}_{2}\left(t_{1}, T\right)\right)=2$. Initial conditions, which have to be elements of the bi-dimensional plane $\operatorname{ker}\left(\mathbf{A}_{2}\right)$ and simulaneously satisfy $x_{2}(0)=d$ are underdetermined: there is a continuum of such initial conditions. Accordingly, a free parameter can be chosen to describe this continuum. The interval in which it lives in controlled by the admissibility of the displacements corresponding to this initial condition. Interestingly, the extremal values correspond to cases which are common solutions of the 1-ipp and the 2-ipp problems. Moreover, the two 1-ipp solutions happen to be actually the same solution shifted in time by half a period as depicted in Fig. 13. Such solutions are also illustrated in a cross-section of the phase plot in Fig. 14. The 1-ipp smooth manifold (only partially represented, in blue) gives rise to a surface made of a continuum of 2-ipp orbits. Moreover,

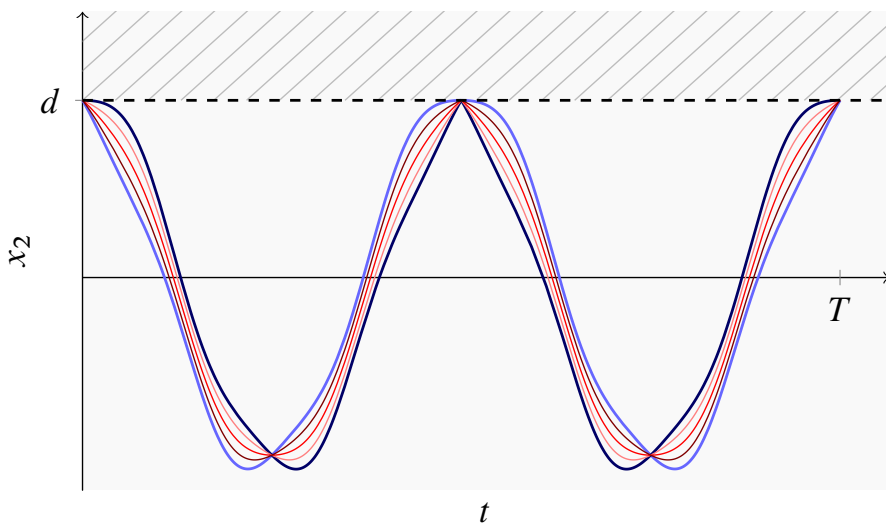

Figure 13. Continuum of 2-ipp orbits of period T ([-], [-] and [-]) limited by one 1-ipp solution of period $T$ [-] and the same 1-ipp solution shifted by T/2 [-]. The 2-ipp solutions differ by the value of the free parameter which determines $\tilde{\mathbf{q}}(0)$ in the kernel of $\mathbf{A}_{2}$.

the solutions contained in the continuum of 2-ipp orbits exhibit all the same period but distinct energies. In other words, the

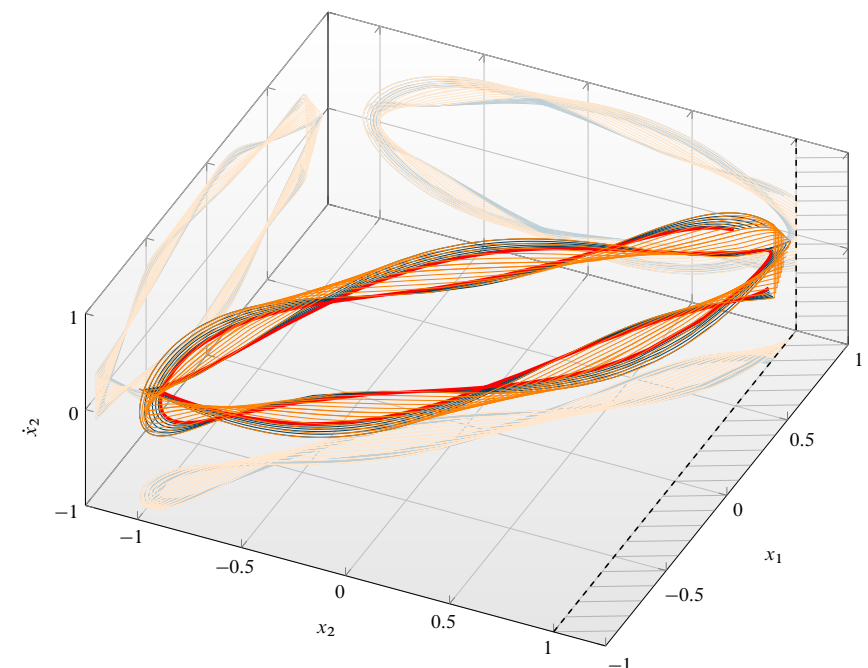

Figure 14. Periodic orbits in the phase portrait $\left(x_{1}, x_{2}, \dot{x}_{2}\right)$ for a period $T$ such that the transition is grazing: 1-ipp [-] and 2-ipp [-] orbits. Grazing solution [-]. 2-ipp orbits all have the same period $T$.

frequency-energy plot in Fig. 8 features a vertical line connecting the branch of 1-ipp admissible solutions and the branch of 2-ipp admissible solutions, zoomed-in in Fig. 15. Let us insist on the

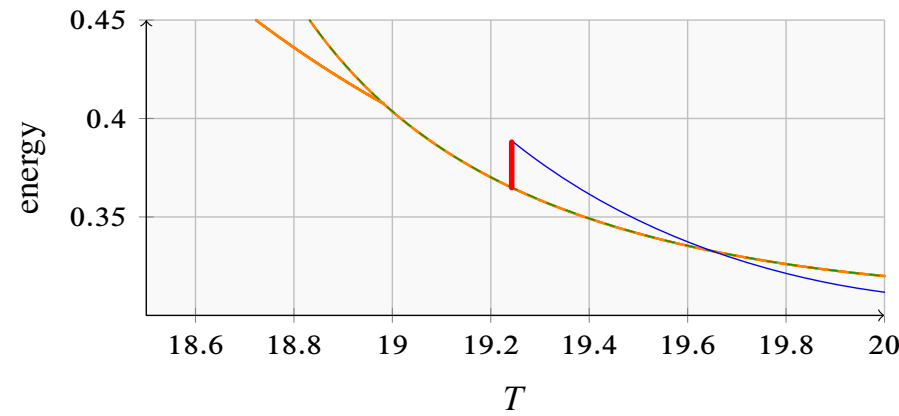

Figure 15. Zones of admissible 1-ipp and 2-ipp solutions. Bridge [-] between the two manifolds. 1-ipp solutions [-] and 2-ipp solutions [-]. Branch of 2-ipp solutions obtained by dilation of the 1-ipp solution branch [-- ]. The height of this graph with the same scale as Fig. 8 would be about the thickness of the curves of Fig. 8.

differences between the orange curves in Fig. 14 and the nonsmooth manifolds in Fig. 10 and 12: the orange curves correspond to trajectories which all have the exact same period and for which the two conditions $x_{2}\left(t_{1}\right)=d$ and $x_{2}(T)=d$ are equivalent. They differ by the value of the free parameter which controls the choice of the initial conditions in the kernel of $\mathbf{A}_{2}$. Nonsmooth modes described previously do not all have the same period; they differ by a parameter which corresponds to a curve on the roots of $\operatorname{det}\left(\mathbf{A}_{2}\right)$.

\section{Conclusion}

The free periodic dynamics of a simple oscillator with two degreesof-freedom undergoing unilateral conditions reflected as a Newton impact law was explored. Its behaviour proved to be extremely rich, dispite its apparent simplicity. A linear mapping $\mathbf{A}_{m}$ which governs the existence of periodic solutions was defined for any number of impacts per period $m$. Continuous families of periodic orbits emerge from this mapping for $m=1$ and $m=2$ 
and were used to define the concept of nonsmooth modes of vibration. The frequency of these orbits is driven by the natural frequencies of the underlying linear system. Such modes were illustrated, and open doors to several key improvements in the analysis of nonsmooth systems.

For $m=1$, the admissibility of these trajectories is controlled by three kinds of transitions: singular, linear modal or grazing. The latter merges solutions with different numbers of impacts per period. This was illustrated for one and two impacts per period but is not fully understood yet. Configurations with $m \geqslant 3$ seem to be only degenerate counterparts of solutions featuring fewer impacts per period.

From preliminary numerical experiments, the results with two degrees-of-freedom extend to larger systems. This sounds promising for a systematic nonsmooth modal analysis, including modal reduction of more challenging nonsmooth systems. Next steps include mathematical proofs of the conjectures presented here as well as stability analyses.

\section{References}

[1] R.M. RosenberG. "On nonlinear vibrations of systems with many degrees of freedom". Advances in applied mechanics 9 1966, 155. DOI: $10.1016 / \mathrm{S} 0065-2156$ (08) 70008-5.

[2] S.W. SHAW, C. PIERRE. "Non-linear normal modes and invariant manifolds". Journal of Sound and Vibration 150(1) 1991, 170-173. DOI: $10.1016 / 0022-460 \mathrm{X}$ (91) 90412-D.

[3] S.W. SHAW, C. PIERRE. "Normal modes for non-linear vibratory systems". Journal of sound and vibration 164(1) 1993, 85-124. DOI: $10.1006 / \mathrm{jsvi} .1993 .1198$.

[4] A.F. Vakakis, L.I. Manevitch, Y.V. Mikhlin, V.N. Pilipchuk, A.A. ZEVIN. Normal modes and localization in nonlinear systems. Springer, 1996. DOI: $10.1002 / 9783527617869$.

[5] G. Kerschen, M. Peeters, J.-C. Golinval, A.F. Vakakis "Nonlinear normal modes, part I: a useful framework for the structural dynamicist". Mechanical Systems and Signal Processing 23(1) 2009, 170-194. DOI: $10.1016 / \mathrm{j} \cdot \mathrm{ymssp} .2008 .04 .002$.

[6] S.-L. CHEN, S.W. SHAW. "Normal modes for piecewise linear vibratory systems". Nonlinear Dynamics 10(2) 1996, 135-164. DOI: $10.1007 / \mathrm{BF} 00045454$.

[7] M. Chati, R. RAND, S. MukHERJEe. "Modal analysis of a cracked beam". Journal of Sound and Vibration 207(2) 1997, 249-270. DOI: 10.1006/jsvi.1997.1099.

[8] D. JiAng, C. PiERRE, S.W. ShaW. "Large-amplitude non-linear normal modes of piecewise linear systems". Journal of sound and vibration 272(3) 2004, 869-891. DOI: $10.1016 / \mathrm{S} 0022-460 X$ (03) 00497-8.

[9] V. ACARY, B. BRogliato. Numerical methods for nonsmooth dynamical systems: applications in mechanics and electronics. Volume 35. Springer, 2008.

DOI: $10.1007 / 978-3-540-75392-6$.
[10] O. BRÜLS, V. ACARY, A. CARDONA. "Simultaneous enforcement of constraints at position and velocity levels in the nonsmooth generalized- $\alpha$ scheme". Computer Methods in Applied Mechanics and Engineering $2812014,131-161$.

DOI: $10.1016 / \mathrm{j} . \mathrm{cma} .2014 .07 .025$.

OAI: hal-01059823.

[11] S.F. MAsri, Y.A. Mariamy, J.C. Anderson. "Dynamic response of a beam with a geometric nonlinearity". Journal of Applied Mechanics 48(2) 1981, 404-410. DOI: $10.1115 / 1.3157630$.

[12] D.J. WAGG, S.R. BISHOP. "Application of non-smooth modelling techniques to the dynamics of a flexible impacting beam". Journal of Sound and Vibration 256(5) 2002, 803-820. DOI: $10.1006 / \mathrm{j}$ svi.2002.5020.

[13] C.-H. Lamarque, O. JANIN. "Modal analysis of mechanical systems with impact non-linearities: limitations to a modal superposition". Journal of Sound and Vibration 235(4) 2000, 567-609. DOI: 10.1006/jsvi.1999.2932.

[14] V.N. PILIPCHUK. "Impact modes in discrete vibrating systems with rigid barriers". International Journal of Non-Linear Mechanics 36(6) 2001, 999-1012.

DOI: $10.1016 / \mathrm{S} 0020-7462(00) 00066-4$.

[15] M. PASCAL. "Dynamics and stability of a two degree of freedom oscillator with an elastic stop". Journal of Computational and Nonlinear Dynamics 1(1) 2005, 94-102. DOI: $10.1115 / 1.1961873$.

[16] D. LAXALDE, M. LEGRAND. "Nonlinear modal analysis of mechanical systems with frictionless contact interfaces". Computational Mechanics 47(4) 2011, 469-478. DOI: $10.1007 / \mathrm{s} 00466-010-0556-3$. OAI: hal-00492775v2.

[17] E.H. Moussi, D. Bellizzi, B. Cochelin, I. Nistor. "Nonlinear normal modes of a two degrees-of-freedom piecewise linear system" 2013. OAI: hal-00783088.

[18] G.-W. LUO, J.-H. XIE. "Hopf bifurcation of a two-degree-offreedom vibro-impact system". Journal of Sound and Vibration 213(3) 1998, 391-408. DOI: $10.1006 /$ jsvi.1997.1361.

[19] D.J. WAGG. "Periodic sticking motion in a two-degree-of-freedom impact oscillator". International Journal of Non-Linear Mechanics 40(8) 2005, 1076-1087. DOI: $10.1016 / j$.ijnonlinmec. 2005.03.002.

[20] M. JEAN, V. ACARY, Y. MONERIE. "Non-smooth contact dynamics approach of cohesive materials". Philosophical Transactions of the Royal Society of London. Series A: Mathematical, Physical and Engineering Sciences 359(1789) 2001, 2497-2518. DOI: $10.1098 /$ rsta.2001.0906.

[21] P. BALlard. "The dynamics of discrete mechanical systems with perfect unilateral constraints". Archive for Rational Mechanics and Analysis 154(3) 2000, 199-274. DOI: $10.1007 / \mathrm{s} 002050000105$. OAI: hal-00111308. 